\title{
ESTUDIO ETOLÓGICO DEL Sylvilagus brasiliensis, DEL BOSQUE SECO TROPICAL DEL DEPARTAMENTO DEL TOLIMA (COLOMBIA)*
}

\author{
Román D. Castañeda ${ }^{1}$ Lina M. Peñuela ${ }^{1}$, Carlos E. Viana ${ }^{2}$
}

\section{Resumen}

El comportamiento del Sylvilagus brasiliensis en el bosque seco tropical del departamento del Tolima no ha sido estudiado, de esta manera el objetivo de este trabajo de investigación fue evaluar el comportamiento de los Sylvilagus en condiciones de cautiverio. Fueron utilizados dos lepóridos, los cuales estaban alojados en corrales individuales ambientados a las condiciones naturales. Los datos se tomaron durante 5 días consecutivos, en intervalos de 5 minutos, con 1 minuto de observación a través de cámaras de video, identificando las variables: descansar, dormir, dar vueltas, roer, beber, comer, orinar, defecar, cecotrofía, acicalar. Se observó que el Sylvilagus brasiliensis presenta hábitos de comportamiento nocturnos, debido al alto porcentaje de actividades realizadas en este periodo, y durante el día los animales se dedican a descansar y a dormir.

Palabras clave: conejos silvestres, comportamiento en cautiverio, lepóridos.

\section{ETHOLOGICAL STUDY OF Sylvilagus brasiliensis IN THE TROPICAL DRY FOREST IN TOLIMA DEPARTMENT - COLOMBIA}

\begin{abstract}
The behavior of Sylvilagus brasiliensis in the tropical dry forest of the department of Tolima, has not been studied. The objective of this study was to evaluate the behavior of Sylvilagus in captivity. They were used two Leporidae which were housed in natural conditions acclimated to individual pens. Data were collected for five consecutive days at intervals of five minutes, with one minute of observation through video cameras, identifying variables rest, sleep, tossing and turning, crack, drinking, eating, urinating, defecating, cecotrophy and grooming. We found that Sylvilagus brasiliensis have nocturnal habits behavior, due to the high percentage of activities in this period, and during the day the animals were devoted to rest and sleep.
\end{abstract}

Key words: behavior in captivity, leporidae, wild rabbits

\footnotetext{
* FE: 14-XI-2014, FR: 27-IV-2015

${ }^{1}$ Profesor asistente - Facultad de Medicina Veterinaria y Zootecnia - Grupo de Investigación en Sistemas Agroforestales Pecuarios, Universidad del Tolima. Ibagué, Colombia. E-mail: rcastaneda@ut.edu.com

${ }^{2}$ Decano Facultad de Medicina Veterinaria y Zootecnia - Grupo de Investigación IMPRONTA - Universidad Cooperativa de Colombia - Sede Ibagué.
} 


\section{INTRODUCCIÓN}

El conocimiento del comportamiento y manejo de los conejos facilita el mejoramiento de ciertos parámetros productivos, al disminuir el estrés. Dentro de los lagomorfos se encuentra el Sylvilagus brasiliensis (Figura 1), que se caracteriza fenotípicamente por tener tamaño mediano, con un peso que varía de 700 a 1000 g; la coloración del pelo es marrón amarillenta, aunque se encuentran individuos de color rojizo oscuro; la zona ventral es blanquecina y en el dorso del cuello tienen un color rojizo (EISENBERG \& REDFORD, 1999). La distribución geográfica del $S$. brasiliensis comprende el sur del estado de Tamaulipas en México, pasando por la Costa Atlántica, hasta el sur de Brasil; zonas caracterizadas por bosques tropicales, selvas y pastizales cercanos a bosques templados. CLARENCE (1996) reporta que estos lepóridos no se ubican en grandes altitudes, exceptuando la península de Yucatán, y la parte suroccidental de Sudamérica; aunque en Colombia han sido encontrados en ecosistemas de alta montaña como el Parque Nacional Natural Los Nevados (INSUASTY et al., 2008).

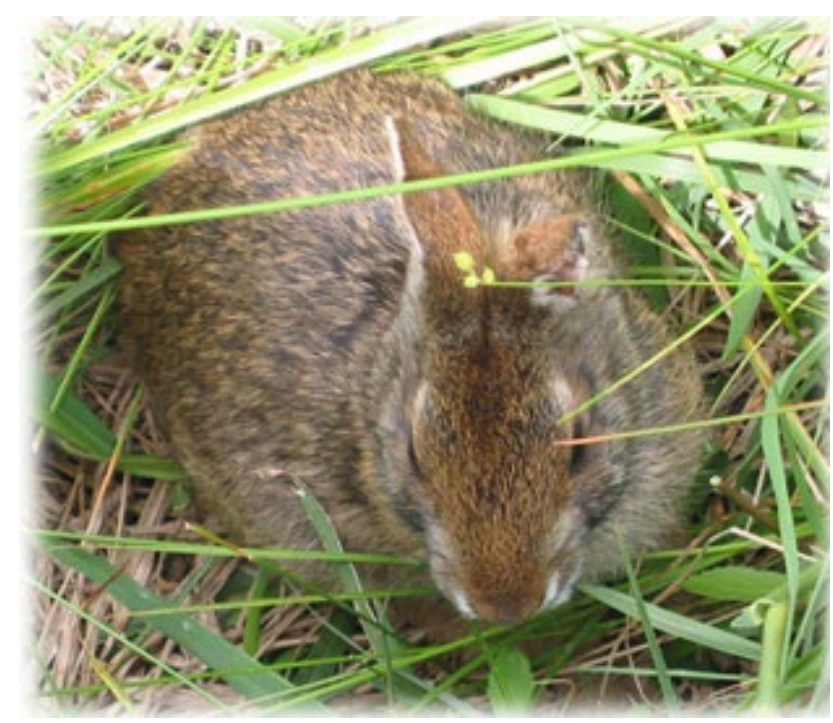

Figura 1. Sylvilagus brasiliensis en su hábitat natural.

Los conejos, al estar ubicados en la parte inferior de la cadena trófica, son presa de diferentes depredadores tales como aves, cánidos, mustélidos y félidos; razón por la cual presentan hábitos nocturnos (16:00 y 8:00), evitando ser identificados durante el día por sus depredadores (MAFFEI et al., 2002). Al ser herbívoros y específicamente folívoros, su dieta se basa exclusivamente de gramíneas (VALERO \& DURANT, 2001); sin embargo, pueden variar tanto temporal como espacialmente (COPERRINDER et al., 1986).

El comportamiento reproductivo de los conejos silvestres suele caracterizarse por existir un macho por cada 2 hembras, pero da la casualidad de que el macho 
dominante es quien se reproduce con las hembras del grupo, permitiendo solo oler y mirar a los demás machos. Estas actividades están sujetas a los cambios de la duración de la luz solar, ya que los conejos perciben estos cambios de luz, disminuyendo la actividad reproductiva debido a que la relacionan con la llegada del invierno (CAMPS, 1997).

La presencia de los conejos en zonas rurales permite obtener beneficios para las diferentes poblaciones que allí se ubican, debido a que la carne de conejo es una fuente de proteína; al tener más claridad en su comportamiento se puede llegar a mejorar parámetros productivos que se traducen en mayor oferta de proteína para las poblaciones, que mejoran la alimentación de las mismas; aunque, se deben realizar más investigaciones debido a que es poca la información disponible (BURESH, 2004).

\section{MATERIALES Y MÉTODOS}

\section{Área de estudio}

El trabajo fue realizado en el bosque seco tropical del departamento del Tolima, entre las coordenadas geográficas: 401'06,58" Norte - 74058'04.31" Oeste y 4³3'56,91" Norte - 74º57'7,89" Oeste. La región se caracteriza por tener temperaturas superiores a $\operatorname{los} 24^{\circ} \mathrm{C}$; precipitaciones bimodales durante el año, inferiores a $1400 \mathrm{~mm}$, con mala distribución y alta evaporación; generándose regímenes de humedad secos y subhúmedos. Altura sobre el nivel del mar de 250 a $700 \mathrm{~m}$. El bosque seco tropical se encuentra en la zona central del departamento, bordeando el río Magdalena de sur a norte; donde se incluyen zonas agroecológicas con una biofísica homogénea de piso térmico cálido, y una extensión de 424,97 $\mathrm{km}^{2}$, con coberturas ralas a densas de pastos, que incluyen áreas de por lo menos 30 municipios del departamento (CHAMORRO \& GALLO, 1998).

\section{Muestreo y análisis}

La recolección de muestras fue realizada a través de trampeos en transectos determinados con jaulas tipo Tomahawk, también fueron realizados muestreos utilizando una red directamente en las madrigueras de los animales. Fueron capturados 2 lepóridos, los cuales fueron llevados al campus de la Universidad Cooperativa de Colombia - sede Ibagué, donde se realizó un examen clínico de cada animal, encontrándose todos en buenas condiciones de salud. Posteriormente, fueron introducidos en corrales ambientados para su adaptación.

\section{Metodología}

La metodología empleada para la toma de datos fue ubicar los conejos en corrales que tuvieron las siguientes dimensiones: largo $2,70 \mathrm{~m}$ por $1,23 \mathrm{~m}$ de ancho y 0,71 m de alto; construidos en ladrillo, cemento y techado con teja Eternit, recreando un ambiente similar a las condiciones naturales (Figura 2). 


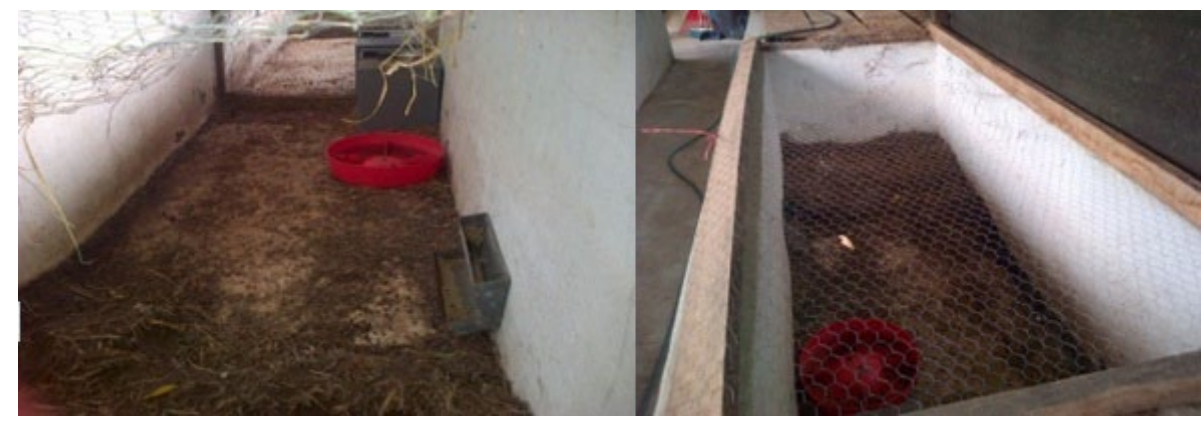

Figura 2. Corrales experimentales donde se observaron los Syvilagus.

Las condiciones ambientales (temperatura y humedad relativa) en los corrales, durante el periodo de muestreo fueron:

Tabla 1. Varias climáticas (temperatura y humedad relativa) medidas durante el periodo experimental.

\begin{tabular}{ccccc}
\hline Día & & Temperatura $\left({ }^{\circ} \mathrm{C}\right)$ & & Humedad relativa (\%) \\
& Máxima & Mínima & Promedio & \\
\hline 1 & 43 & 16,4 & 28,9 & 50 \\
2 & 37,1 & 16,6 & 29,2 & 37 \\
3 & 37,3 & 19,4 & 27 & 35 \\
4 & 37,3 & 16,6 & 26 & 68 \\
5 & 37 & 16 & 24,3 & 64 \\
\hline
\end{tabular}

Los datos se tomaron a 2 conejos Sylvilagus brasiliensis (un macho y una hembra); durante 5 días consecutivos, en intervalos de 5 minutos, con 1 minuto de observación a través de cámaras de video para un total de 120 horas. Las variables medidas fueron: dar vueltas, beber, comer, cecotrofía, roer, dormir, orinar, defecar, descansar, acicalar.

\section{Análisis estadístico}

Los resultados fueron analizados utilizando estadística descriptiva, estimando el porcentaje de cada una de las variables de estudio en los dos individuos, dividiendo en dos periodos (Día - Noche) e intervalos de 1 hora.

\section{RESULTADOS}

Los datos obtenidos del análisis de comportamiento del Sylvilagus brasiliensis, durante el periodo de muestreo se presentan a continuación: 


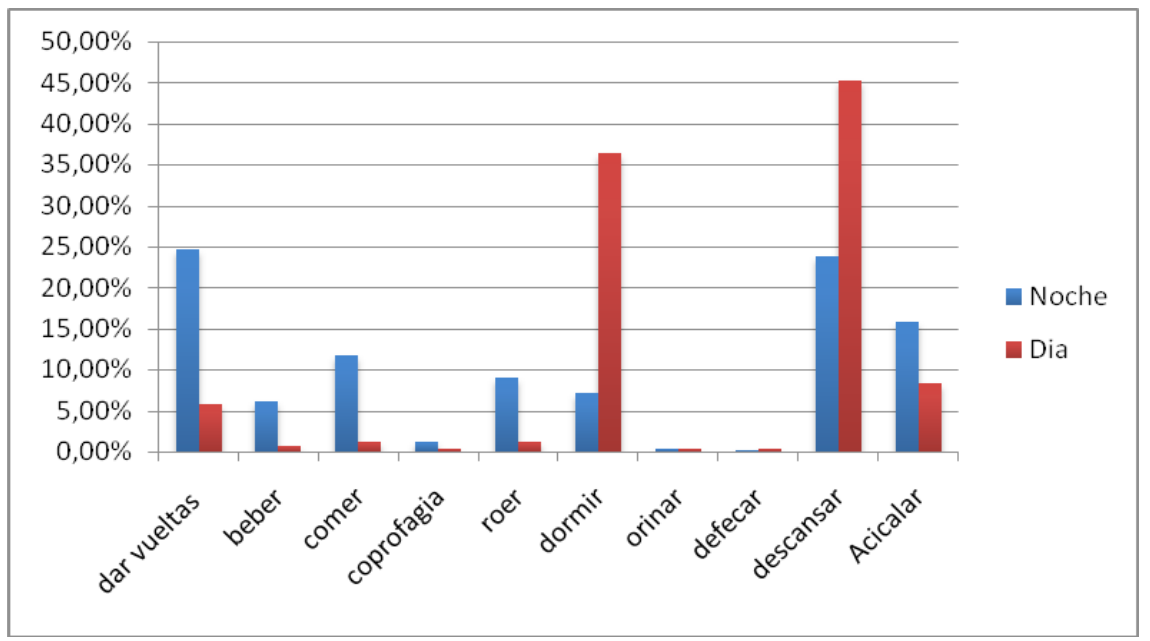

Figura 3. Porcentaje de actividades realizadas Día vs Noche en Sylvilagus mantenidos en condiciones de cautiverio.

En el periodo Día (6:00 am a 6:00 pm) se caracterizan por tener un alto porcentaje en actividades que emplean poco movimiento o desplazamiento, como lo son descansar (45,28\%) y dormir (36,39\%); seguido de actividades como acicalar (8,33\%), dar vueltas $(5,83 \%)$, roer $(1,25 \%)$, comer $(1,11 \%)$, beber $(0,69 \%)$, cecotrofía $(0,42 \%)$, defecar $(0,42 \%)$, orinar $(0,28 \%)$. A diferencia del periodo anterior, en el periodo Noche (6:00 pm a 6:00 am) se caracterizan por tener un alto porcentaje en actividades de búsqueda, reconocimiento y alimentación, como lo son dar vueltas $(24,72 \%)$, descansar $(23,75 \%)$, acicalar $(15,83 \%)$, comer $(11,81 \%)$, seguido de actividades como roer $(9,03 \%)$, dormir $(7,22 \%)$, beber $(6,11 \%)$, cecotrofía $(1,11 \%)$, orinar $(0,28 \%)$, defecar $(0,14 \%)$.

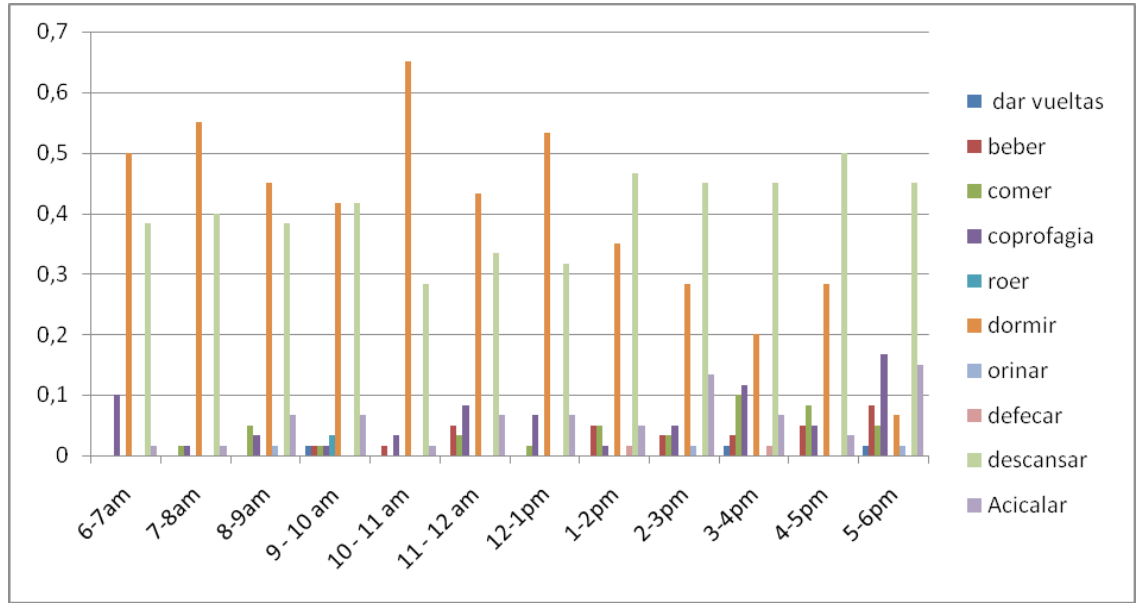

Figura 4. Porcentaje de actividades realizadas durante el Día (6 am - 6 pm) en Sylvilagus mantenidos en condiciones de cautiverio. 
Las principales variables que presentan mayores valores porcentuales durante el Día son: dormir (65\%) 10-11 am y descansar (50\%) 4-5 pm. Seguido de ellas se encuentran: coprofagia (17\%) 5-6 pm, acicalar (15\%) 5-6 pm, comer (10\%) 3-4 pm, beber (8\%) 5-6 pm; las restantes variables presentan un valor inferior al $8 \%$.

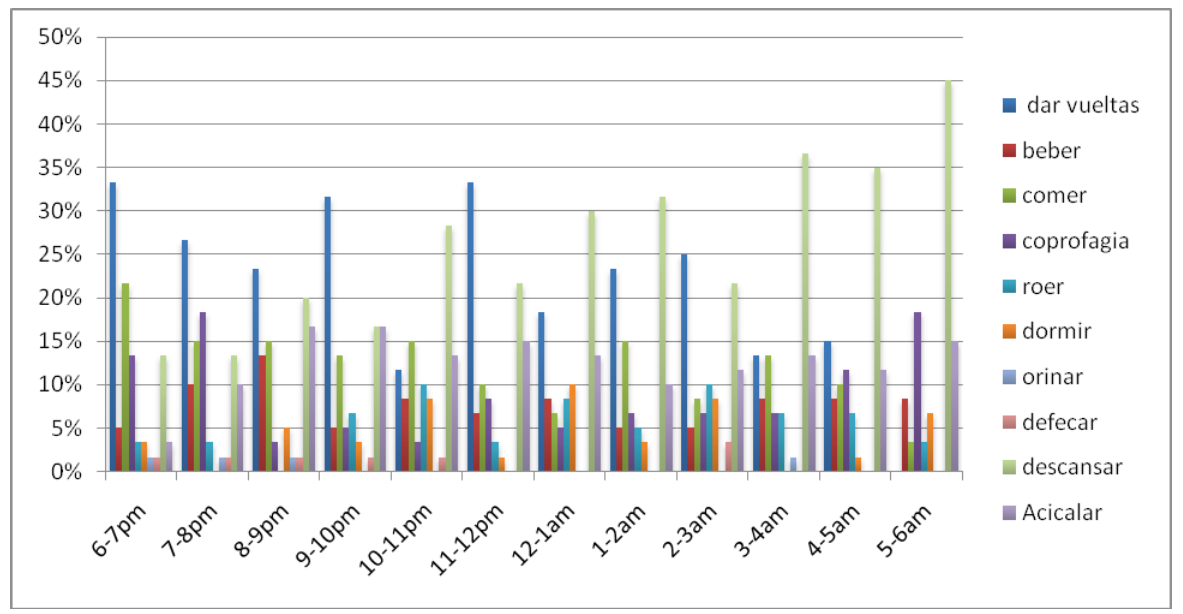

Figura 5. Porcentaje de actividades realizadas durante la Noche (6 pm - 6 am) en Sylvilagus mantenidos en condiciones de cautiverio.

Durante el periodo de Noche los valores porcentuales de las diferentes variables son: descansar (45\%) 5-6 am, dar vueltas (33\%) 6-7 pm y 11-12 pm, comer (22\%) 6-7 pm, acicalar (17\%) 8-9 pm, beber (13\%) 8-9 pm, dormir (10\%) 12-1 am, roer (10\%) $10-11 \mathrm{pm}$ y 2-3 pm; las restantes variables presentan un valor inferior al $10 \%$.

\section{DISCUSIÓN}

Dentro de las variables, que se tuvieron en cuenta para conocer el comportamiento del Sylvilagus brasiliensis, se evidencia que los conejos dedican gran parte de su tiempo a dormir y descansar durante el periodo día, lo cual está relacionado con lo reportado por MAFFEI et al. (2002), los cuales observaron que las especies del genero Sylvilagus tienen hábitos nocturnos (04:00 pm a 8:00 am) realizando en estas horas actividades como comer, desplazarse y roer.

DURANT \& PÉREZ (1988) mencionan que el Sylvilagus brasiliensis al ser presa de depredados como aves, cánidos, mustélidos y félidos, toma como mecanismo de defensa el disminuir sus actividades durante el día; de igual forma, el conejo descansa en el día evitando las altas temperaturas que aumentarían su temperatura corporal (SAMOGGIA, 1985).

La variables "dar vueltas" y "roer" se relacionan entre sí, ya que es un mecanismo de reconocimiento del conejo y de respuesta al cautiverio, probablemente en su hábitat natural los Sylvilagus no tengan este tipo de comportamiento. Las dos variables presentan un mayor porcentaje durante el periodo Noche con respecto al periodo Día, y confirman que estos individuos se alimentan en las horas de la 
noche, donde tienen más probabilidades de sobrevivir a sus predadores ya que durante el día pueden ser identificados fácilmente (GONZÁLEZ-REDONDO, 2006).

Las variables "beber" y "comer" son dos variables que aumentan durante el periodo Noche, debido al mecanismos de defensa para evadir la identificación por parte de sus depredadores (XU, 1996); y al comportamiento nictameral del conejo silvestre (GUTIÉRREZ et al., 2006), ya que en los domesticados el comportamiento de comer y beber cambia, llevándose a cabo durante las 24 horas del día, con una leve tendencia a un aumento en la noche (LEBAS et al., 1996 en GUTIÉRREZ et al., 2006).

La cecotrofía no presenta diferencias y se relaciona con lo mencionado por XU (1996), quien encontró que no existen diferencias significativas en cuanto al consumo de cecótrofos durante el día o la noche; aunque hay una leve tendencia a consumirse más durante las horas de la noche.

El consumo de alimento se lleva a cabo terminando la tarde y durante la noche aumenta, las partículas finas van entrando y fermentando en el ciego, hasta que ocurre el vaciamiento en las horas de la mañana (CARABAÑO \& MERINO, 1996), dando origen a los cecótrofos. Durante la cecotrofía, se disminuye el consumo de alimento y cesa la excreción de heces duras (DE BLAS et al., 2002), lo cual explica el comportamiento alimenticio en el periodo nocturno. Asi mismo, los lepóridos presentan defecan en un mayor porcentaje en el periodo Día, debido a que el tránsito digestivo del conejo dura en promedio 20 horas (FAO, 1986).

\section{CONCLUSIONES}

Se concluye que los lepóridos silvestres del género Sylvilagus en el Tolima tienen hábitos de comportamiento nocturnos, donde dedican la mayor parte del tiempo a alimentarse y dar vueltas, debido al efecto del cautiverio. Entre tanto, durante el día los Sylvilagus se dedican a dormir y a descansar en un $80 \%$.

\section{BIBLIOGRAFÍA}

BURESH, J., 2004.- Sylvilagus brasiliensis. Animal Diversity Web. Recuperado de http://animaldiversity. ummz.umich.edu/accounts/Sylvilagus_brasiliensis/

CAMPS, J., 1997.- Importancia de la etología para mejorar el manejo de los conejos. Cunicultura, 22 (129): 247-251.

CARABAÑO, R. \& MERINO, J.M., 1996.- Effect of ileal cannulation on feed intake, soft and hard faecs excretion throughout the day in rabbits: 121-126 (en) Proceedings 6th World Rabbit Congress. Toulouse.

CHAMORRO, D. \& GALLO, J.E., 1998.- Gramíneas y Leguminosas. El Poira, Ibagué.

CLARENCE, M.F., 1996.- Manual Merck de Veterinaria. Editora Roca, São Paulo.

COPERRINDER, A., BOYD, R.J. \& STUART, H.R., 1986.- Inventory and monitoring of wildlife habitat. Service Center, Denver.

DE BLAS, J.C., GARCÍA, J. \& CARABAÑO, R., 2002.- Avances en nutrición de conejos. Revisión a las principales peculiaridades del sistema digestivo de los conejos, que son responsables, entre otras causas, de la complejidad de esta producción. Boletín de Cunicultura, 122: 6-16.

DURANT, P. \& PÉREZ R., 1988.- Distribución geográfica y ecológica de los conejos silvestres de Venezuela. El conejo de páramo y el conejo de monte. Monog., CDCHT-ULA. Mérida, Venezuela.

EISENBERG, J. \& REDFORD, K., 1999.- Mammals of the Neotropics. The Central Neotropics. Ecuador, Peru, Bolivia, Brazil. Chicago and London: The University of Chicago Press.

FAO, 1986.- El conejo: cría y patología. Organización de las Naciones Unidas para la Agricultura y la Alimentación. Recuperado de http://www.fao.org/docrep/014/t1690s/t1690s02.pdf

GONZÁLEZ-REDONDO, P., 2006.- Viabilidad de la producción intensiva del conejo de monte en pureza. Ganadería, 38: 50-54. 
GUTIÉRREZ, R.P., SAUQUILLO, J. \& TORRES, A., 2006.- La iluminación en las granjas cunícolas (1ra Parte). Boletín de Cunicultura, 145: 6-11.

INSUASTY OSORIO, J.A., RAMÍREZ BENAVIDES, G.F. \& MEJÍA MORALES, L.M., 2008.- Estudio epidemiológico del conejo silvestre Sylvilagus brasiliensis en el parque nacional natural los nevados. Bol. cient. mus. hist. nat., 12, 90-110.

MAFFEI, L., CUÉLLAR, E. \& NOSS, A., 2002.- Uso de trampas-cámara para la evaluación de mamíferos en el ecotono Chaco-Chiquitanía. Revista Biología Ecología, 11: 55-65.

SAMOGGIA, G., 1985.- Actitudes y comportamiento del conejo. Bol. Cunicult., 72.

VALERO, L. \& DURANT, P., 2001.- Análisis de la dieta del conejo de páramo, S. brasiliensis meridensis Thomas, 1904 (Lagomorpha: Leporidae) en Mucubaji, Mérida, Venezuela. Revista de Ecología Latino Americana, 8 (2): 1-13.

XU, H.T., 1996.- El comportamiento del conejo. Cunicultura: 329-332. 